\title{
THE JOINT SPECTRUM OF COMMUTING NONNORMAL OPERATORS
}

\begin{abstract}
JOHN BUNCE ${ }^{1}$
Abstracr. We define the joint approximate spectrum of a finite family of bounded commuting operators on a Hilbert space and show that it is closed and nonempty. If the operators are all hyponormal, then the family need not be finite, and the joint approximate spectrum can be defined in terms of the multiplicative linear functionals on the generated $C^{*}$-algebra. These definitions extend the usual definition of the joint spectrum of a family of commuting normal operators.
\end{abstract}

Let $S$ be a set of operators in $B(H)$, the set of bounded operators on a Hilbert space $H$. Let $C^{*}(S)$ be the $C^{*}$-subalgebra of $B(H)$ generated by the set $S$ and the identity $I$. By a character on $C^{*}(S)$ we mean a multiplicative linear functional $\rho$ such that $\rho(I)=1$. Let $\operatorname{sp}(A)$ denote the spectrum of an operator $A$ in $B(H)$, and let $p(A)$ denote the set of eigenvalues of $A$. We denote by $a(A)$ the approximate point spectrum of $A$; i.e., $a(A)$ is the set of scalars $\lambda$ for which there is a sequence $\left(x_{n}\right)$ of unit vectors in $H$ such that $(A-\lambda I) x_{n}$ converges to zero in norm. If $A$ is a normal operator then $a(A)=\operatorname{sp}(A)$ (see [5] for facts about spectra). If $S=\left\{A_{1}, \cdots, A_{n}\right\}$ is a commuting family of normal operators, then the joint spectrum of the $\left\{A_{1}, \cdots, A_{n}\right\}$ is defined as the set of $n$-tuples of scalars $\left(\lambda_{1}, \cdots, \lambda_{n}\right)$ such that the ideal of $C^{*}(S)$ generated by the set $\left\{A_{i}-\lambda_{i} I: i=1, \cdots, n\right\}$ is different from $C^{*}(S)[6,3.1 .13]$. It follows from the Gelfand-Neumark representation theorem for commutative $C^{*}$-algebras that the joint spectrum of a family of commuting normal operators is nonempty $[6,3.1 .14]$. In this note we show that a set called the joint approximate spectrum can be defined for any finite family of commuting operators, and we show that if all the operators are hyponormal (i.e., $A A^{*} \leqq A^{*} A$ ) then the joint approximate spectrum can be defined in terms of the characters on the $C^{*}$-algebra generated by the family of operators, just as is the case for normal operators.

In $[2$, Corollary 3] it was shown that $a(A)=\{\lambda: B(H)(A-\lambda I)$

Received by the editors June 22, 1970.

AMS 1969 subject classifications. Primary 4755, 4730; Secondary 4665.

Key words and phrases. Joint spectrum, approximate spectrum, joint approximate spectrum, hyponormal operators.

${ }^{1}$ The author held an AFOSR-NRC postdoctoral fellowship while writing this paper.

Copyright @ 1971, American Mathematical Society 
$\neq B(H)\}$. We will of ten use the fact that every maximal left ideal of $B(H)$ is of the form $K(\rho)=\left\{A \in B(H): \rho\left(A^{*} A\right)=0\right\}=\{A \in B(H)$ : $\rho(D A)=0$ for all $D \in B(H)\}$ for some pure state $\rho$ on $B(H)[3,2.9 .5]$. Any state $\rho$ on $B(H)$ induces a representation $\pi_{\rho}$ of $B(H)$ which acts on a Hilbert space $H_{\rho}$ and has canonical cyclic vector $\xi_{\rho}$. We use freely facts about this representation (see [3]).

Proposition 1. Let $A_{1}, A_{2}, \cdots, A_{n}$ be operators in $B(H)$ such that $A_{i} A_{j}=A_{j} A_{i}$ for all $i$ and $j$. If $\lambda_{1}, \lambda_{2}, \cdots, \lambda_{n-1}$ are scalars such that

$$
B(H)\left(A_{1}-\lambda_{1} I\right)+\cdots+B(H)\left(A_{n-1}-\lambda_{n-1} I\right) \neq B(H),
$$

then there is a scalar $\lambda_{n}$ such that

$$
B(H)\left(A_{1}-\lambda_{1} I\right)+\cdots+B(H)\left(A_{n}-\lambda_{n} I\right) \neq B(H) .
$$

PRoof. The proper left ideal $B(H)\left(A_{1}-\lambda_{1} I\right)+\cdots+B(H)\left(A_{n-1}-\lambda_{n-1} I\right)$ is contained in a maximal left ideal $K(\rho)$ of $B(H)$. Then it is easily seen that $\pi_{\rho}\left(A_{i}\right) \xi_{\rho}=\lambda_{i} \xi_{\rho}$ for $1 \leqq i \leqq n-1$. Let $L$ $=\left\{x \in H_{\rho}:\left(\pi_{\rho}\left(A_{i}\right)-\lambda_{i} I\right) x=0,1 \leqq i \leqq n-1\right\}$. The subspace $L$ is nonzero and clearly invariant under $\pi_{\rho}\left(A_{i}\right)$ for $1 \leqq i \leqq n$. Let $\lambda_{n}$ be any scalar in $a\left(\pi_{\rho}\left(A_{n}\right) \mid L\right)$. Then if $I_{L}$ is the identity in $B(L), I_{L}$ does not belong to $B(L)\left(\pi_{\rho}\left(A_{n}\right) \mid L-\lambda_{n} I_{L}\right)$. Suppose, for contradiction, that $B(H)=B(H)\left(A_{1}-\lambda_{1} I\right)+\cdots+B(H)\left(A_{n}-\lambda_{n} I\right)$. Then there exist $D_{i} \in B(H), 1 \leqq i \leqq n$, such that $I=D_{1}\left(A_{1}-\lambda_{1} I\right)+\cdots+D_{n}\left(A_{n}-\lambda_{n} I\right)$. Let $P$ be the projection of $H_{\rho}$ onto $L$. Then using the fact that $\left(\pi_{\rho}\left(A_{i}\right)-\lambda_{i} I\right) \mid L=0$ for $1 \leqq i \leqq n-1$, we get that

$$
I_{L}=P \pi_{\rho}\left(D_{n}\right) \mid L\left(\pi_{\rho}\left(A_{n}\right) \mid L-\lambda_{n} I_{L}\right)
$$

which contradicts our choice of $\lambda_{n}$. Hence $B(H) \neq B(H)\left(A_{1}-\lambda_{1} I\right)$ $+\cdots+B(H)\left(A_{n}-\lambda_{n} I\right)$ and the proposition is proved. We note that in Proposition 1 we have $\lambda_{i} \in a(A)$ for $1 \leqq i \leqq n$.

Definition 1. For $A_{1}, A_{2}, \cdots, A_{n}$ commuting operators in $B(H)$, let the joint approximate spectrum of the set $\left\{A_{1}, A_{2}, \cdots, A_{n}\right\}$ be the set $\left\{\left(\lambda_{1}, \cdots, \lambda_{n}\right): B(H)\left(A_{1}-\lambda_{1} I\right)+\cdots+B(H)\left(A_{n}-\lambda_{n} I\right)\right.$ $\neq B(H)\}$ and denote this set by $a\left(A_{1}, A_{2}, \cdots A_{n}\right)$.

Since $a\left(A_{1}\right)$ is nonempty, using Proposition 1 inductively immediately shows that $a\left(A_{1}, \cdots, A_{n}\right)$ is nonempty and is in fact a subdirect product of the product of the $a\left(A_{j}\right)$; that is, the projection of $a\left(A_{1}, \cdots, A_{n}\right)$ on the $j$ th-coordinate space is onto $a\left(A_{j}\right)$ for each $j$. Proposition 1 also shows that if $F$ is any subset of $\{1,2, \cdots, n\}$, then $a\left(\left\{A_{j}: j \in F\right\}\right)$ is the image of $a\left(A_{1}, \cdots, A_{n}\right)$ under the natural map.

Proposition 2. Let $A_{1}, A_{2}, \cdots, A_{n}$ be commuting operators, then $a\left(A_{1}, \cdots, A_{n}\right)$ is a nonempty compact set. 
Proof. We need only show that $a\left(A_{1}, \cdots, A_{n}\right)$ is closed. So suppose $\left(\lambda_{1}^{\alpha}, \lambda_{2}^{\alpha}, \cdots, \lambda_{n}^{\alpha}\right) \in a\left(A_{1}, \cdots, A_{n}\right)$ for $\alpha$ in some index set and $\lim \lambda_{i}^{\alpha}=\lambda_{i}$ for $1 \leqq i \leqq n$. Suppose, for contradiction, that $\left(\lambda_{1}, \cdots, \lambda_{n}\right)$ $\notin a\left(A_{1}, \cdots, A_{n}\right)$. Then

$$
B(H)=B(H)\left(A_{1}-\lambda_{1} I\right)+\cdots+B(H)\left(A_{n}-\lambda_{n} I\right),
$$

so there exist operators $D_{1}, D_{2}, \cdots, D_{n}$ in $B(H)$ such that $I=D_{1}\left(A_{1}-\lambda_{1} I\right)+\cdots+D_{n}\left(A_{n}-\lambda_{n} I\right)$. Then

$$
\begin{aligned}
\left\|I-\left(D_{1}\left(A_{1}-\lambda_{1}^{\alpha} I\right)+\cdots+D_{n}\left(A_{n}-\lambda_{n}^{\alpha} I\right)\right)\right\| \\
\quad=\left\|\left(\lambda_{1}^{\alpha}-\lambda_{1}\right) D_{1}+\cdots+\left(\lambda_{n}^{\alpha}-\lambda_{n}\right) D_{n}\right\| \\
\quad \leqq\left\|D_{1}\right\|\left|\lambda_{1}^{\alpha}-\lambda_{1}\right|+\cdots+\left\|D_{n}\right\|\left|\lambda_{n}^{\alpha}-\lambda_{n}\right| .
\end{aligned}
$$

Hence for large enough $\alpha$, we have

$$
\left\|I-\left(D_{1}\left(A_{1}-\lambda_{1}^{\alpha} I\right)+\cdots+D_{n}\left(A_{n}-\lambda_{n}^{\alpha} I\right)\right)\right\|<1 .
$$

But then $D_{1}\left(A_{1}-\lambda_{1}^{\alpha} I\right)+\cdots+D_{n}\left(A_{n}-\lambda_{n}^{\alpha} I\right)$ is an invertible operator which contradicts the fact that $\left(\lambda_{1}^{\alpha}, \cdots, \lambda_{n}^{\alpha}\right) \in a\left(A_{1}, \cdots, A_{n}\right)$. Hence $a\left(A_{1}, \cdots, A_{n}\right)$ is closed.

We recall that an operator $A$ is called hyponormal if $A A^{*} \leqq A^{*} A$.

Proposition 3. Let $A_{1}, A_{2}, \cdots, A_{n}$ be commuting hyponormal operators. Then for each $\left(\lambda_{1}, \cdots, \lambda_{n}\right) \in a\left(A_{1}, \cdots, A_{n}\right)$ there is a pure state $\rho$ on $B(H)$ such that $\rho\left(A_{j}\right)=\lambda_{j}$ for $1 \leqq j \leqq n$ and $\rho(C D)=\rho(C) \rho(D)$ for all $C \in B(H)$ and all $D \in C^{*}\left(\left\{A_{1}, \cdots, A_{n}\right\}\right)$. In particular $\rho$ is a character on $C^{*}\left(\left\{A_{1}, \cdots, A_{n}\right\}\right)$.

Proof. If $\left(\lambda_{1}, \cdots, \lambda_{n}\right) \in a\left(A_{1}, \cdots, A_{n}\right)$ then $J=B(H)\left(A_{1}-\lambda_{1} I\right)$ $+\cdots+B(H)\left(A_{n}-\lambda_{n} I\right)$ is a proper left ideal of $B(H)$, so there is a pure state $\rho$ on $B(H)$ such that $J \subset K(\rho)=\left\{D \in B(H): \rho\left(D^{*} D\right)=0\right\}$. Now since $A_{j}$ is hyponormal,

$$
\left(A_{j}-\lambda_{j} I\right)\left(A_{j}-\lambda_{j} I\right)^{*} \leqq\left(A_{j}-\lambda_{j} I\right)^{*}\left(A_{j}-\lambda_{j} I\right)
$$

and by a well-known result of R. G. Douglas [4], we have that there is a bounded operator $D_{j}$ on $H$ such that $D_{j}\left(A_{j}-\lambda_{j} I\right)=\left(A_{j}-\lambda_{j} I\right)^{*}$ or $B(H)\left(A_{j}-\lambda_{j} I\right)^{*} \subset B(H)\left(A_{j}-\lambda_{j} I\right)$. Now $K(\rho) \subset \rho^{-1}(0)$ so for each $C$ in $B(H)$ we have $\rho\left(C\left(A_{j}-\lambda_{j} I\right)\right)=\rho\left(C\left(A_{j}-\lambda_{j} I\right)^{*}\right)=0$ for each $j$. Hence $\rho\left(C A_{j}\right)=\lambda_{j} \rho(C)=\rho(C) \rho\left(A_{j}\right)$ and $\rho\left(C A_{j}^{*}\right)=\bar{\lambda}_{j} \rho(C)=\rho(C) \rho\left(A_{j}^{*}\right)$ for each $j$. Now $\left\{D \in B(H): \rho(C D)=\rho(C) \rho(D)\right.$ and $\rho\left(C D^{*}\right)=$ $\rho(C) \rho\left(D^{*}\right)$ for all $\left.C \in B(H)\right\}$ is clearly a $C^{*}$-algebra which contains $A_{j}$ for each $j$. So $\rho(C D)=\rho(C) \rho(D)$ for all $D \in C^{*}\left(\left\{A_{1}, \cdots, A_{n}\right\}\right)$ and all $C \in B(H)$. Also $\rho\left(A_{j}\right)=\lambda_{j}$ for each $j$.

The following corollary generalizes Corollary 11 of [2]. 
Corollary 4. If $A_{1}, A_{2}, \cdots, A_{n}$ are commuting hyponormal operators, then $a\left(A_{1}, \cdots, A_{n}\right)=\left\{\left(\rho\left(A_{1}\right), \cdots, \rho\left(A_{n}\right)\right): \rho\right.$ is a character on $\left.C^{*}\left(\left\{A_{1}, \cdots, A_{n}\right\}\right)\right\}$, and if

$$
J=\bigcap\left\{\rho^{-1}(0): \rho \text { is a character on } C^{*}\left(\left\{A_{1}, \cdots, A_{n}\right\}\right)\right\},
$$

then

$$
C^{*}\left(\left\{A_{1}, \cdots, A_{n}\right\}\right) / J \cong C\left(a\left(A_{1}, \cdots, A_{n}\right)\right) .
$$

Proof. If $\rho$ is a character on $C^{*}\left(\left\{A_{1}, \cdots, A_{n}\right\}\right)$, let $\rho_{0}$ be any extension of $\rho$ to a state on $B(H)[3,2.10,1]$. Then

$$
\rho\left(\left(A_{j}-\rho\left(A_{j}\right) I\right)^{*}\left(A_{j}-\rho\left(A_{j}\right) I\right)\right)=0
$$

for each $j$, so $A_{j}-\rho\left(A_{j}\right) I \in K\left(\rho_{0}\right)$ for each $j$, and $K\left(\rho_{0}\right)$ is a proper left ideal of $B(H)$. Combining this with the definition of $a\left(A_{1}, \cdots, A_{n}\right)$ and Proposition 3, we see that $a\left(A_{1}, \cdots, A_{n}\right)=\left\{\left(\rho\left(A_{1}\right), \cdots, \rho\left(A_{n}\right)\right)\right.$ : $\rho$ is a character on $\left.C^{*}\left(\left\{A_{1}, \cdots, A_{n}\right\}\right)\right\}$. Now $C^{*}\left(\left\{A_{1}, \cdots, A_{n}\right\}\right) / J$ is clearly a commutative $C^{*}$-algebra generated by the normal operators $A_{j}+J$, so by $[6,3.1 .15], C^{*}\left(\left\{A_{1}, \cdots, A_{n}\right\}\right) / J \cong$ $C(X)$, where $X=\left\{\left(\rho\left(A_{1}+J\right), \cdots, \rho\left(A_{n}+J\right)\right): \rho\right.$ a character on $\left.C^{*}\left(\left\{A_{1}, \cdots, A_{n}\right\}\right) / J\right\}$. Now if $\pi: C^{*}\left(\left\{A_{1}, \cdots, A_{n}\right\}\right) \rightarrow$ $C^{*}\left(\left\{A_{1}, \cdots, A_{n}\right\}\right) / J$ is the canonical projection, then $\rho \rightarrow \rho \circ \pi$ is a bijection between the characters of $C^{*}\left(\left\{A_{1}, \cdots, A_{n}\right\}\right) / J$ and $C^{*}\left(\left\{A_{1}, \cdots, A_{n}\right\}\right)$. Hence $X=a\left(A_{1}, \cdots, A_{n}\right)$.

The ideal $J$ in Corollary 4 is the commutator ideal of $C^{*}\left(\left\{A_{1}, \cdots, A_{n}\right\}\right)$; that is, $J$ is the closed two-sided ideal generated by elements of the form $D^{*} D-D D^{*}$ with $D \in C^{*}\left(\left\{A_{1}, \cdots, A_{n}\right\}\right)$. We note that Corollary 4 implies that our definition of joint approximate spectrum coincides with the usual definition of joint spectrum for commuting families of normal operators and implies that the joint spectrum of commuting normal operators is a subdirect product of the product of the individual spectra, a fact which we have not seen in the literature. We now extend the definition of joint approximate spectrum to arbitrary families of commuting hyponormal operators.

Proposition 5. Let $S=\left\{A_{\alpha}: \alpha \in \Lambda\right\}$ be a commuting family of hyponormal operators. If $A_{\alpha_{1}}, \cdots, A_{\alpha_{n}}$ is a finite subset of $S$ and $\left(\lambda_{1}, \cdots, \lambda_{n}\right) \in a\left(A_{\alpha_{1}}, \cdots, A_{\alpha_{n}}\right)$, then there exists a pure state $\rho$ on $B(H)$ such that $\rho\left(A_{\alpha_{j}}\right)=\lambda_{j}$ for $1 \leqq j \leqq n$ and $\rho(C D)=\rho(C) \rho(D)$ for all $C \in B(H)$ and $D \in C^{*}(S)$. In particular $\rho$ is a character on $C^{*}(S)$.

Proof. Let $N=$ all finite subsets of $\Lambda$ which contain $\left\{\alpha_{1}, \cdots, \alpha_{n}\right\}$ and direct $N$ by set inclusion. Then by the proof of Proposition 3 and the discussion after Definition 1, for each $F \in N$ there is a pure 
state $\rho_{F}$ on $B(H)$ such that $\rho_{F}\left(A_{\alpha_{j}}\right)=\lambda_{j}$ for $1 \leqq j \leqq n$ and $\rho_{F}(C D)=$ $\rho_{F}(C) \rho_{F}(D)$ for all $C \in B(H)$ and $D \in C^{*}\left(\left\{A_{\alpha}: \alpha \in F\right\}\right)$. Now the set of states on $B(H)$ is weak*-compact, so there is a state $\rho_{0}$ on $B(H)$ which is a cluster point of the net $\left\{\rho_{F}: F \in N\right\}$. Let $C \in B(H)$ and $D \in \cup\left\{C^{*}\left(\left\{A_{\alpha}: \alpha \in F\right\}\right): F \in N\right\}$, then there is a $F_{0} \in N$ such that $D \in C^{*}\left(\left\{A_{\alpha}: \alpha \in F_{0}\right\}\right)$. If $F_{0} \subset F$, then $\rho_{F}(C D)=\rho_{F}(C) \rho_{F}(D)$ and hence $\rho_{0}(C D)=\rho_{0}(C) \rho_{0}(D)$. It then follows that for all $C \in B(H)$ and all $\alpha \in \Lambda, \quad \rho_{0}\left(C A_{\alpha}\right)=\rho_{0}(C) \rho_{0}\left(A_{\alpha}\right) \quad$ and $\rho_{0}\left(C A_{\alpha}^{*}\right)=\rho_{0}(C) \rho_{0}\left(A_{\alpha}^{*}\right)$. Thus $\rho_{0}\left(C\left(A_{\alpha}-\rho_{0}\left(A_{\alpha}\right) I\right)\right)=\rho_{0}\left(C\left(A_{\alpha}^{*}-\rho_{0}\left(A_{\alpha}^{*}\right) I\right)\right)=0$ for all $C \in B(H)$, so $A_{\alpha}-\rho_{0}\left(A_{\alpha}\right) I$ and $A_{\alpha}^{*}-\rho_{0}\left(A_{\alpha}^{*}\right) I$ are in $K\left(\rho_{0}\right)$ for all $\alpha \in \Lambda$. Let $\rho$ be a pure state on $B(H)$ such that $K\left(\rho_{0}\right) \subset K(\rho)$. Then for all $\alpha \in \Lambda$, $\rho\left(C A_{\alpha}\right)=\rho(C) \rho\left(A_{\alpha}\right)$ and $\rho\left(C A_{\alpha}^{*}\right)=\rho(C) \rho\left(A_{\alpha}^{*}\right)$, hence $\rho(C D)=\rho(C) \rho(D)$ for all $C \in B(H)$ and $D \in C^{*}(S)$. It is clear that $\rho\left(A_{\alpha_{j}}\right)=\lambda_{j}$ for $1 \leqq j \leqq n$.

For a family $S=\left\{A_{\alpha}: \alpha \in \Lambda\right\}$ of commuting hyponormal operators, let $a\left(A_{\alpha}: \alpha \in \Lambda\right)=\left\{\left(\rho\left(A_{\alpha}\right)\right)_{\alpha \in \Lambda}: \rho\right.$ is a character on $\left.C^{*}(S)\right\}$. We call $a\left(A_{\alpha}: \alpha \in \Lambda\right)$ the joint approximate spectrum of the $\left\{A_{\alpha}: \alpha \in \Lambda\right\}$. By Proposition $5, a\left(A_{\alpha}: \alpha \in \Lambda\right)$ is nonempty and is a subdirect product of the product of the $a\left(A_{\alpha}\right)$. In fact $a\left(A_{\alpha}: \alpha \in \Lambda\right)$ is the inverse limit of the sets $a\left(A_{\alpha}: \alpha \in F\right)$ for $F$ a finite subset of $\Lambda$. Thus $a\left(A_{\alpha}: \alpha \in \Lambda\right)$ is also a compact set. Let $J=\left\{\rho^{-1}(0): \rho\right.$ a character on $\left.C^{*}(S)\right\}$ be the commutator ideal of $C^{*}(S)$. Then just as in Corollary 4 , we have that $C^{*}(S) / J \cong C\left(a\left(A_{\alpha}: \alpha \in \Lambda\right)\right)$. We remark that an example of a commuting family of hyponormal operators is a one-parameter semigroup of isometries.

We now use Proposition 3 to give some information about the approximate point spectrum of a single hyponormal operator.

Proposition 6. Let $A=U P$ be the polar decomposition of a hyponormal operator $A$. If $\lambda \in a(A)$ then $|\lambda| \in a(P)$ and if $\lambda \neq 0$ then $\arg (\lambda) \in a(U)$.

Proof. Since $\|A x\|=\|P x\|$ for all $x$ in the Hilbert space, $0 \in a(A)$ if and only if $0 \in a(P)$. So we suppose $0 \neq \lambda \in a(A)$. Then by Proposition 3 there is a pure state $\rho$ on $B(H)$ such that $\rho(A)=\lambda$ and $\rho(C D)$ $=\rho(C) \rho(D)$ for all $C \in B(H)$ and $D \in C^{*}(A)$. Now $P=\left(A^{*} A\right)^{1 / 2}$ $\in C^{*}(A)$, so $\rho(A)=\rho(U P)=\rho(U) \rho(P)=\lambda$. Also $U^{*} A=P$, so $\rho(P)$ $=\rho\left(U^{*}\right) \rho(A)$ and $\rho(A)=|\rho(U)|^{2} \rho(A)$. Hence $|\rho(U)|=1$ and $|\lambda|$ $=\rho(P), \arg (\lambda)=\rho(U)$. Now $\rho$ is a character on $C^{*}(A)$ and $C^{*}(P)$ $C C^{*}(A)$, so $\rho$ is a character on $C^{*}(P)$ and by [2, Proposition 8] this implies $|\lambda|=\rho(P) \in a(P)$. Also $U^{*} A=U^{*} U P=P$, so $\rho(P)=\rho\left(U^{*} U\right) \rho(P)$ and $\rho\left(U^{*} U\right)=1$. Then it is easily seen that

$$
\rho\left((U-\rho(U))^{*}(U-\rho(U))\right)=0,
$$


so $U-\rho(U) \in K(\rho), B(H)(U-\rho(U)) \neq B(H)$ and $\rho(U)=\arg (\lambda) \in a(U)$. Note that we did not actually use the fact that $U P$ was the polar decomposition of $A$; we needed only that $P \geqq 0$ and that $U$ was a partial isometry whose initial space contained the range of $P$ such that $A=U P$.

An operator $A$ with polar decompsition $U P$ is called quasinormal if $U$ and $P$ commute. These operators were studied (under another name) by Arlen Brown [1]. Quasinormal operators are also hyponormal and in this case Proposition 6 can be improved:

Proposition 7. Let $A$ be a quasinormal operator with polar decomposition UP. Then if $\alpha \in \operatorname{sp}(P)$, there is a $z \in a(U)$ such that $z \alpha \in a(A)$; and if $z \in a(U)$, there is $a \alpha \in \operatorname{sp}(P)$ such that $z \alpha \in a(A)$.

Proof. It is easily seen that the partial isometry in the polar decomposition of a hyponormal operator is itself hyponormal, so $U$ and $P$ are commuting hyponormal operators and $C^{*}(A) \subset C^{*}(\{U, P\})$. If $(z, \alpha) \in a(U, P)$, then by Proposition 3 there is a character $\rho$ on $C^{*}(\{U, P\})$ such that $\rho(U)=z$ and $\rho(P)=\alpha$. Then $\rho(A)=\rho(U) \rho(P)$ $=z \alpha$ and $\rho(A) \in a(A)$ since $\rho$ is a character on $C^{*}(A)$. Proposition 7 is then immediate since $a(U, P)$ is a subdirect product of $a(U)$ and $a(P)$. Note that we used only the fact that $A$ was a product of the commuting hyponormals $U$ and $P$.

If $K$ is a Hilbert space let $\tilde{K}$ be the set of sequences $\left(x_{0}, x_{1}, \cdots\right)$ with $x_{i} \in K$ for all $i$ and the sequence $\left(\left\|x_{i}\right\|\right)$ square-summable. Define a bounded operator $S$ on $\tilde{K}$ by $S\left(x_{0}, x_{1}, \cdots\right)=\left(0, x_{0}, x_{1}, \cdots\right)$. If $P$ is a positive operator on $K$, define a bounded operator $\tilde{P}$ on $\tilde{K}$ by $\tilde{P}\left(x_{0}, x_{1}, \cdots\right)=\left(P x_{0}, P x_{1}, \cdots\right)$ and let $\hat{P}=S \tilde{P}$. Then $S$ and $\tilde{P}$ are commuting hyponormals, $\tilde{P} \geqq 0$ and $S^{*} \hat{P}=\widetilde{P}$. If $P$ is one-to-one, then $S \tilde{P}$ is the polar decomposition of $\hat{P}$. We call the quasinormal operator $\hat{P}$ the dilated shift operator defined by $P$. Every quasinormal operator $A$ is unitarily equivalent to one of the form $\hat{P} \oplus C$ for $C$ some normal operator [1].

Proposition 8. Let $\hat{P}$ be the dilated shift operator defined by the positive operator $P$. Then $a(\hat{P})=\{z \alpha:|z|=1, \alpha \in \operatorname{sp}(P)\}$.

Proof. First we show that if $\lambda \in a(\hat{P})$ then $z \lambda \in a(\hat{P})$ for all $|z|=1$; that is, $a(\hat{P})$ has circular symmetry. Let $x^{n}=\left(x_{0}^{n}, x_{1}^{n}, \cdots\right) \in \tilde{K}$ be a sequence such that $\left\|x^{n}\right\|=1$ for all $n$ and such that $\left\|\hat{P} x^{n}-\lambda x^{n}\right\| \rightarrow 0$. Then for $z$ of modulus one let $y^{n} \in \tilde{K}$ be given by $y_{i}^{n}=z^{i} x_{i}^{n}, i=0,1$, $2, \cdots$. That is, $y^{n}=\left(x_{0}^{n}, z x_{1}^{n}, z^{2} x_{2}^{n}, \cdots\right)$. Then $\left\|y^{n}\right\|=1$ and a calculation shows that 


$$
\left\|(\hat{P}-\bar{z} \lambda) y^{n}\right\|^{2}=\left\|(\hat{P}-\lambda) x^{n}\right\|^{2} \rightarrow 0 .
$$

Hence $\bar{z} \lambda \in a(\hat{P})$ and $a(\hat{P})$ has circular symmetry. It is clear that $\operatorname{sp}(P)=\operatorname{sp}(\widetilde{P})$ and it is well known that $a(S)=\{z:|z|=1\}$. Propositions 6 and 7 and the remarks at the end of their proofs then imply that $a(\hat{P})=\{z \alpha:|z|=1, \alpha \in \operatorname{sp}(P)\}$.

We remark that James A. Deddens has noted that everything in the open ball of radius $\|P\|$ is an eigenvalue for the adjoint of $\hat{P}$ and consequently the spectrum of $\hat{P}$ is the closed ball of radius $\|P\|$.

We close by noting that if $A_{1}$ and $A_{2}$ are hyponormal and $A_{1} A_{2}$ $=A_{2} A_{1}$, then $a\left(A_{1}, A_{2}\right)$ may be much smaller than the product of $a\left(A_{1}\right)$ and $a\left(A_{2}\right)$. For example let $K$ be the space of square-summable complex sequences, let $U$ be the unilateral forward shift, $U\left(x_{0}, x_{1}, \cdots\right)=\left(0, x_{0}, x_{1}, \cdots\right)$. Then let $H=K \oplus K$ and let $A_{1}$ $=U \oplus 0$ and $A_{2}=0 \oplus U$. Then $a\left(A_{1}\right)=a\left(A_{2}\right)=\{\lambda:|\lambda|=1\} \cup\{0\}$. But $a\left(A_{1}, A_{2}\right)$ equals the set $\{(\lambda, 0):|\lambda|=1\} \cup\{(0, \lambda):|\lambda|=1\}$.

ADDED IN PROOF. Proposition 6 of this paper is essentially Lemma 7 in C. R. Putnam's paper, An inequality for the area of hyponormal spectra, Math. Z. 116 (1970), 323-330.

\section{REFERENCES}

1. Arlen Brown, On a class of operators, Proc. Amer. Math. Soc. 4 (1953), 723-728. MR 15, 538.

2. J. Bunce, Characters on single generated $C^{*}$-algebras, Proc. Amer. Math. Soc. 25 (1970), 297-303.

3. Jacques Dixmier, Les $C^{*}$-algèbres et leurs représentations, Cahiers Scientifiques, fasc. 29, Gauthier-Villars, Paris, 1964. MR 30 \#1404.

4. R. G. Douglas, On majorization, factorization, and range inclusion of operators on Hilbert space, Proc. Amer. Math. Soc. 17 (1966), 413-415. MR 34 \#3315.

5. Paul R. Halmos, Introduction to Hilbert space, Chelsea, New York, 1957.

6. Lars Hörmander, An introduction to complex analysis in several variables, Van Nostrand, Princeton, N. J., 1966. MR 34 \#2933.

University of Kansas, Lawrence, Kansas 66044 